\title{
ESTRATEGIAS DE MARKETING PARA LA GENERACIÓN DE MICROEMPRESA ESCOLAR EN EL CENTRO EDUCATIVO TÉCNICO POLITÉCNICO REGIONAL ÁREA CARPINTERÍA
}

\author{
Escobar Vicuña, Teodorico Pablo y Chávez Pajares, Marcos Antonio
}

Facultad de Administración de la Universidad Nacional del Centro del Perú

\begin{abstract}
RESUMEN
El presente trabajo de investigación tiene por finalidad alcanzar una propuesta organizativa, adecuada al proceso de enseñanza-aprendizaje que los estudiantes del Centro Educativo Técnico Politécnico Regional del área de carpintería tengan y apliquen los conocimientos adquiridos en clase y que contribuya a la generación de microempresas no solo en la rama de la carpintería, sino también en cualquier área técnica bajo un trabajo digno que les puede servir de sustento económico y de éxito profesional. Dicho estudio esta en concordancia con el proceso conducente a la formación integral del estudiante. La perspectiva actual exige estar inmersos en este camino, puesto que, otros países están bajo este enfoque y más aun porque los estudiantes serán los directamente beneficiados en este nuevo cambio que indudablemente se va a traducir en calidad en todos los aspectos y consecuentemente profesionales que coadyuven al desarrollo de la región. Se trata de una investigación descriptiva, que se agencia de bibliografías actualizadas, de encuestas y entrevistas en relación al contexto de la microempresa escolar. El presente trabajo tiene por objetivo formular las estrategias del marketing que generen la microempresa escolar en el Centro Educativo Técnico "Politécnico Regional" del área de carpintería. Se realiza un diagnóstico a través del análisis FODA que nos permitió identificar las fortalezas y debilidades que tiene el proyecto, de igual modo, conocer las oportunidades y amenazas que se debe de enfrentar en un mercado tan competitivo. Los resultados más importantes que es necesario enfatizar, consideramos: proponer un sistema de producción moderno basado en la calidad del acabado y del diseño. Con una visión, misión y valores integral y sea asumida y practicada por todos los integrantes. La investigación se ha dividido en 4 capítulos que deben desarrollarse en un periodo de un año, finalizando en Marzo del 2009.
\end{abstract}

\section{MARKETING STRATEGIES FOR THE GENERATION OF SCHOOL MICRO COMPANY IN THE CENTRO EDUCATIVO TÉCNICO “POLITÉCNICO REGIONAL” CARPENTRY AREA.}

\begin{abstract}
The present investigation work has for purpose to reach an organizational proposal, appropriate to the process of teaching - learning that the students of the Centro Educativo Técnico "Politécnico Regional" carpentry area have and apply the acquired knowledge in class and that it contributes to the generation of non alone Micro Companies in the branch of the carpentry and also in any technical area under a worthy work that can serve them as economic sustenance but of professional success. This study is in agreement with the conducive process to the student's integral formation. The current perspective demands to be obedient on this road, since, other countries follow this focus and because the students will be those directly beneficiaries in this new change that undoubtedly will transform into quality in all aspects and consequently professionals that contribute to the development of the region. It is about a descriptive investigation that you agency of up-to-date bibliographies, of surveys and interviews in relation to the context of the school micro company. The present study has for objective to formulate the strategies of marketing that school micro company generates in the Centro Educativo Técnico Politécnico Regional carpentry area. It was carried out a diagnosis through the FODA analysis that allowed us identify the strengths and weaknesses the project has, on a same way, to know the opportunities and threats to face in such a competitive market. The most important results that it is necessary to emphasize, consider: to propose a modern production system based on quality and design. With a vision, mission and integral values and to be assumed and practiced by all the members. The investigation has been divided in 4 chapters that should be developed in a year period, ending in March 2009.
\end{abstract}

\footnotetext{
* Este trabajo de investigación fue recibido el 20/03/2009 retornado para su revisión 26/10/2009 y aprobado para su publicación 16/11/2009

1 Email: Ibazan2@hotmail.com

2Email: mgranados@uncp.edu.pe
} 


\section{INTRODUCCIÓN}

El presente trabajo de investigación está orientado a contribuir con la generación de una microempresa escolar en el colegio Politécnico Regional del Centro, como parte de su formación integral que recibe el estudiante, al mismo tiempo generar los ingresos necesarios para poder cubrir los gastos que afronta el colegio y a los mismos estudiantes, dado que son jóvenes de escasos recursos económicos.

Si bien es cierto se tiene ciertas debilidades como la inexperiencia de los estudiantes y la falta de responsabilidad de ellos, pero sobra el optimismo, la fe, la fortaleza, el empeño y las ganas de sobresalir y consideramos que eso es crucial para poder tener éxito en la vida.

Los aspectos legales para la creación de esta microempresa en un colegio estatal existen, sin embargo, puede funcionar como una institución educativa que produce a pequeña escala y como parte de su formación desde luego está exenta de los tributos que se pagan.

La puesta en funcionamiento de una microempresa escolar establecería el inicio de un cambio profundo en la educación del país en el aspecto formativo técnico, por el hecho de que la educación universitaria nacional se ve cada vez mas restringida en el mercado laboral y muchos universitarios están ocupando labores propias a otras carreras 0 están subempleados.

Esta realidad nos lleva a formular que la educación técnica debe priorizarse por los responsables de la educación del Perú.

Las estrategias de marketing según Morrisey, define a la estrategia como la dirección en la que una empresa necesita avanzar para cumplir con su misión.

También se define como el patrón de los movimientos de la organización y de los enfoques de la dirección para lograr los objetivos planteados.
De acuerdo a estas definiciones vemos a la estrategia como un proceso para lograr los objetivos que nos planteamos en el éxito de la microempresa escolar, proponiendo alternativas de producción y venta que sean atractivos y competitivos, considerando al producto, distribución, precio, publicidad y equipo de venta.

\section{MATERIALES Y MÉTODOS}

\section{MATERIALES Y EQUIPOS}

Se agenció de libros, revistas, memorias, documentos sustentatorios, folletos y como equipo una computadora con Internet.

\section{MÉTODO DE INVESTIGACIÓN}

El método que se esta desarrollando es el deductivo - inductivo, puesto que, a partir de las experiencias y todo el conocimiento que se tiene adquirido vamos a plasmar e inducir a nuestra problemática.

\section{DISEÑO METODOLÓGICO}

Se trata de una investigación descriptiva, puesto que, se diseñará un conjunto de estrategias que permitirán un mejor aprovechamiento de los conocimientos y práctica impartidas en el taller de carpintería del Centro Educativo Técnico Politécnico Regional a través de la generación de la Microempresa Escolar.

\section{RESULTADOS}

De la encuesta aplicada y de la entrevista realizada, se muestra que las fortalezas y debilidades, recomiendan un conjunto de estrategias. 


\section{DIAGNÓSTICO DE LA MICROEMPRESA ESCOLAR}

\section{ANÁLISIS FODA}

\section{FORTALEZAS}

- Capital propio.

- Infraestructura propia.

- La calidad de los productos es igual o mejor que otros productores.

- Los recursos humanos son jóvenes y mucho por aprender.

- Innovación en los productos.

- Servicio al cliente de manera directa y personalizada.

- Maquinaria y herramientas propias y suficientes.

- Variedad de productos y bien surtido.

- Hay generación de utilidades todas las semanas.

\section{OPORTUNIDADES}

- Mano de obra joven y barata.

- Exploración de nuevos distritos, buscando nuevos mercados para vender a más gente.

- Su mercado crece de manera rápida, debido al producto barato.

- La población local esta creciendo, por tanto, las ventas puede ser rebasado.

- Desarrollo de tecnología e innovación.

- Participación en licitaciones públicas o privadas.

- Establecimiento de convenios con instituciones para producirles sus bienes de madera.

\section{DEBILIDADES}

- Falta de planeación en la microempresa escolar.

- Falta de caja registradora.

- Ausencia de sistema de seguridad.

- Conocimientos de gestión empírico.

- Falta de responsabilidad de algunos estudiantes.

- Inexperiencia de los estudiantes.

- Falta de cultura organizacional.

- Los productos son vendidos a sus familiares y vecinos.

\section{AMENAZAS}

- Debido al crecimiento de la población, entraran nuevos competidores.

- Existen productos sustitutos por parte de otras empresas y microempresas.

- Empresa estatal, por tanto sujeta a control y fiscalización rigurosa, implicando cierta ineficacia.

- Jóvenes sin DNI, sujetos a fiscalización por parte de la oficina de trabajo.

- Establecimiento de nuevas tecnologías por parte de los mayores competidores.

- Competencia excesiva en el mercado.

- Empresas con mayor capital.

\section{CRUZ DE PORTER DE LA MICROMPRESA ESCOLAR}

\section{PODER DE NEGOCIACIÓN DE} PROVEEDOR:

1. Maderera Ucayali.

2. Maderera

3. Ferretería "El popular"

Factores determinantes

- Calidad de materia prima

- Precios

\section{NUEVOS INGRESANTES:}

1. Industrias Metálicas.

2. Industrias de madera prensada.

3. Familias de carpinteros que se independizan.

Barreras de entrada

- Capital de inversión considerable

- Entrega inmediata

- Diversidad de productos y modelos.

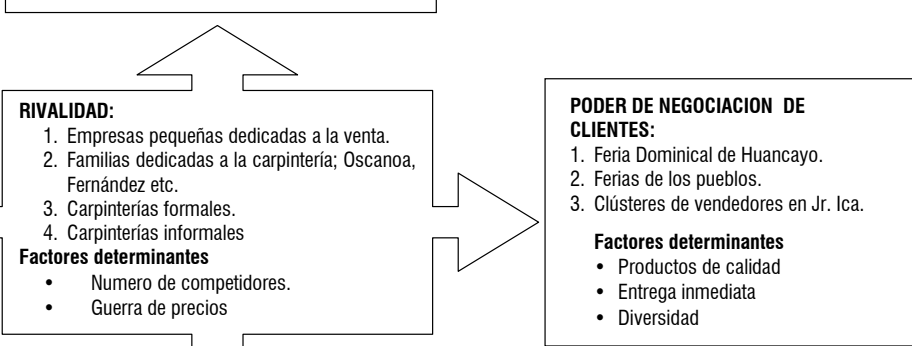


Del análisis del la cadena de valor y de la matriz FODA nos permiten presentar el siguiente resultado.

- Los productos no son vendidos por lo general a posibles clientes potenciales en del mercado, sino se destinan al consumo propio.

- Los productos son producidos en pequeña escala, pudiendo ser producidos de mejor calidad e innovación.

- Los productos pueden ser vendidos a un precio cómodo.

- Desconocen de gestión y comercialización y lo que quiere el mercado.

- Por estar consideraciones podemos sugerir que la implantación de la microempresa escolar estará apoyada por las estrategias de precios y diferenciación.

\section{FORMULACIÓN DE ESTRATEGIAS DE MARKETING PARA LA MICROEMPRESA ESCOLAR}

\section{Modelo a seguir para la generación de una mi- croempresa escolar}

\section{Gastos, ingresos y ganancias.}

La planificación incluye calcular los gastos y los ingresos. Los gastos son determinados por los costos de los materiales, insumos, depreciación, máquinas y mano de obra. Además de los gastos de materiales e insumos, el alumno debe incluir en los gastos sus horas de trabajo considerando que su mano de obra tiene también un costo que influye en el precio del producto, la participación de los estudiantes en el presupuesto del proyecto es parte de su aprendizaje.

\section{Propuesta de estrategias de mercadeo}

El plan de mercadeo es la planificación de la comercialización.

El plan de mercadeo se ocupa de cuatro estrategias básicas para la generación de microempresas escolares.
1 ¿Qué tipo de producto 0 servicio se va a ofertar?

En esta parte la planificación describiendo el producto, determinando en el: su color, tamaño, textura, forma, consistencia, presentación, empaque, etc. Sí queremos dar un curso sobre producción, nuestro plan de mercadeo tendría los siguientes procedimientos: objetivos, contenidos, metodología, materiales impresos, manuales, local, insumos, equipamiento, demanda de clientes, etc.

\section{2 ¿Qué precio tendrá nuestro producto o servicio?}

El proyecto debe considerar y tener presente que la ganancia, que se obtenga como resultado de la venta de los productos, debe ser invertida totalmente en el taller de carpintería. La administración de los ingresos provenientes de la venta de los productos es un aspecto que el jefe de taller debe trabajarlo con sumo cuidado.

Las cuentas deben ser transparentes, de conocimiento de los integrantes del taller. La transparencia en la administración de los recursos, sobre todo, públicos o del equipo es un valor que el maestro debe enfatizar e inculcar permanentemente en los estudiantes.

3 ¿En qué plaza o lugar conviene vender el producto 0 dar el servicio?

El lugar de la venta debe estar cerca de los clientes, debe ser fácil de llegar, confortable para el cliente y bien ubicado donde se muestre fácilmente los productos. El equipo elegirá las mejores condiciones para que los productos o los servicios sean fácilmente apreciados por los sentidos de los clientes.

El estudio de los costos de transporte y la aplicación de adecuadas técnicas de transporte son parte del aprendizaje que deben lograr los miembros del equipo.

4 ¿Cómo hacemos la promoción del producto-servicio?

La promoción de un producto ó servicio debe ser planificada seleccionando y ordenando la informa- 
ción que daremos a los clientes sobre las ventajas del producto 0 del servicio.

Realizar la promoción significa informar y atraer la atención de los clientes para que compren los productos o servicios. Hay varias formas de promocionarlos: La publicidad, que da a conocer con claridad y en pocas palabras las bondades del producto 0 servicio, mediante la radio, la TV, folletos, carteles, paneles, volantes, internet, promoción de ventas, demostraciones, rifas, canjes, reducción de precios, exhibiciones especiales.

\section{ESTRATEGIA DE PRECIOS}

De las encuestas aplicadas y del análisis correspondiente se desprende que el precio de los distintos bienes alcanza el promedio del mercado. La determinación del precio estratégico para alcanzar un precio módico se debe de realizar las compras en grandes volúmenes para así tener un mayor poder de negociación con los proveedores y bajar los costos.

La alianza estratégica se dará con proveedores como Ucayali y Mantaro a través de convenios con estas instituciones de modo que haya una reciprocidad que beneficie a ambas instituciones, dentro de ellos; que la institución educativa envié practicantes del área de carpintería e informática a estos aserraderos, brindar capacitaciones a su personal para un mejor servicio y atención, entre otros. Se espera que los proveedores reduzcan el precio de venta en un $10 \%$.

\section{ESTRATEGIA DE DIFERENCIACIÓN}

\section{Características de la infraestructura}

Se tiene un local amplio que permite que solo falte una distribución ordenada de los materiales y algunas maquinas que se encuentran dispersas, y establecer un ambiente para mostrar los productos acabados.

\section{Maquinaria y equipo}

De acuerdo a las encuestas realizadas se afirma que la institución educativa tiene las suficientes maquina- rias y equipos para poder realizar las tareas, de modo que no se tiene limitaciones para producir productos a gran escala.

\section{Diseño}

Se sugiere producir productos novedosos e innovadores, no producir productos que se encuentran saturados en el mercado. Se requiere producir bienes que sean atractivos para la vista de la gente y dichos diseños están acordes a la modernidad acompañado de la calidad debida.

\section{Acabado}

El acabado, indudablemente uno de los factores por la cual los clientes preferirían el producto, en ese sentido, creemos que el acabado marcará la diferencia para obtener un producto de calidad.

\section{Personal con implementos de seguridad}

Se hace necesario tener implementos de seguridad, puesto que son todavía adolescentes los que trabajan con las maquinas, y en la encuesta realizada ellos sienten ciertos temores por la peligrosidad de las maquinas.

\section{CONCLUSIONES}

- Del análisis FODA y de las encuestas aplicadas se desprende que la infraestructura propia, la maquinaria y equipo completo que se tiene, además del recurso joven, ofrece las fortalezas adecuadas para asegurar el éxito de la microempresa escolar.Sin embargo, la inexperiencia de los alumnos y la falta de una cultura organizacional podrían afectar el desarrollo de la misma.

- De la cruz de Porter, se sintetiza que la rivalidad es fuerte, puesto que, existe un número grande de competidores fabricantes locales, sumados a comerciantes que traen sus productos de Lima, la plaza local y aledaña se torna agresiva. Adicionalmente la entrada de nuevos sustitutos con productos menos costosos y refinados y más maleables se hace competitivo el negocio. 
- Las estrategias de marketing para la generación de la microempresa escolar en el Centro Educativo Técnico Politécnico Regional Área Carpintería, permitirá en los estudiantes el manejo adecuado de sus capacidades intelectuales así como motrices, haciendo que su educación sea integral.

- Del análisis realizado se desprende que la creación de una microempresa escolar en el colegio Politécnico Regional tendrá el éxito esperado, puesto que, se podrá producir productos de buena calidad y a un precio razonable, estableciendo alianzas estratégicas con los proveedores con un descuento del $10 \%$ de los costos.

- Los diseños que se sugieren como productos a generar, tienen las condiciones para que satisfagan a los consumidores, puesto que, son diseños innovadores, elegantes y que fácilmente pueden atraer al cliente.

\section{LITERATURA CITADA}

Stanton, William; Walker, Bruce Etzel, Michel. 1993. Fundamentos de marketing.Novena edición. Mc. Graw. Hill.España.
Kotler, Philip. 2003. Dirección de la mercadotecnia. Séptima edición McGraw.Hill / Interamericana. S.A.México.

Lambin, Jean. 2001. Marketing estratégico. Segunda edición.McGraw. Hill / Interamericana S.A. España.

Avila Acosta Roberto. 1995. Introducción a la metodología de la investigación. Editorial. Concytec. Lima.

Torres Bardales C. 1997. El proyecto de la investigación científica. Editorial. UNMSM. Lima.

Piscoya Hermoza Luís. 1995. Investigación científica y educacional. Editorial. Amaru. Lima.

Porter Michael. 1996. Estrategia competitiva. Compañía Editorial Continental. México.

Berenson, Levine. 2002. Estadística básica en administración. Editorial. Prentice Hall. México. 